\title{
Luby Transform Coding Aided Bit-Interleaved Coded Modulation for the Wireless Internet
}

\author{
R. Y. S. Tee, T. D. Nguyen, S. X. Ng, L-L. Yang and L. Hanzo \\ School of ECS, University of Southampton, SO17 1BJ, UK. \\ http://www-mobile.ecs.soton.ac.uk
}

\begin{abstract}
Bit-Interleaved Coded Modulation using Iterative Decoding (BICM-ID) is amalgamated with Luby Transform (LT) coding. The resultant joint design of the physical and data link layer substantially improves the attainable Bit Error Rate (BER) performance. A Cyclic Redundancy Check (CRC) combined with a novel Log-Likelihood Ratio (LLR) based packet reliability estimation method is proposed for the sake of detecting and disposing of erroneous packets. Subsequently, bit-by-bit LT decoding is proposed, which facilitates a further BER improvement at a lower number of BICM-ID iterations. Finally, we revisit the pseudo random generator function used for designing the LT generator matrix.
\end{abstract}

\section{INTRODUCTION}

The classic data link layer technique of mitigating the wireless channel impairments is to employ Automatic Repeat Requests (ARQ). Naturally, this method requires additional bandwidth for the retransmission of data, once a corrupted packet is received which necessitates a feedback channel. By contrast, in this study we dispense with the feedback channel by jointly designing the physical and data link layer

More explicitly, Luby Transform (LT) [1] coding is employed for eliminating the feedback required by ARQ aided transmissions. LT coding was originally designed for the Binary Erasure Channel (BEC), which requires the transmission of redundant packets for the sake of recovering the original source packets. We propose to amalgamate LT coding with Bit-Interleaved Coded Modulation using Iterative Decoding (BICM-ID) [2] for the sake of mitigating wireless communication channel effects.

The novelty of this paper is that instead of using a simple serial concatenated LT and BICM-ID scheme, we intrinsically amalgamate LT coding and BICM-ID by creating an exchange of extrinsic information between them. A Cyclic Redundancy Check (CRC) is used for detecting the presence of any erroneous packets. A further novel improvement suggested is that an LLR based packet reliability estimation is introduced. Extrinsic Information Transfer (EXIT) charts are used by the proposed LLR-based packet reliability estimation scheme. This potentially enhances the error checking capability, especially at higher Signal to Noise Ratios (SNR) and hence may eliminate the potential redundancy introduced by the CRC overhead.

Error propagation across LT packets encountered during packet decoding may cause failure in LT decoding. Hence, as a counter measure, in this paper we introduce a bit-by-bit LT decoding technique by exploiting the LLR estimations provided by the associated BICM-ID decoder. The advantages are two fold : 1) we reduce the probability of occurrence of erroneous packets which would otherwise have to be discarded. 2) we mitigate the probability of potential error propagation inflicted by erroneous packets. When corrupted bits are in the same index location of difference source packets, we can readily discard them with the aid of LT bit-by-bit decoding as long as the number of received packets contain the minimum number of redundancy. We further propose a novel pseudo-random generator [3] for creating the LT encoded packets, which will allow us to reduce the proportion of redundant packet required for successful LT decoding.

The rest of this contribution is organized as follows. Section II provides an overview of our system, outlining our joint design of

The financial support of the EPSRC, UK, and that of the European Union is gratefully acknowledged.
LT and BICM-ID coding. The proposed CRC and LLR-basd packet reliability estimation scheme designed with the aid of EXIT charts are detailed in Section III. Section IV describes the bit-by-bit LT decoding as well as the pseudo-random LT generator matrix approach. Section V quantifies the achievable performance of this novel jointly design scheme, while our conclusions are presented in Section VI.

\section{SYSTEM OVERVIEW}

The schematic of the joint LT BICM-ID arrangement is shown in Figure 1. Consider a single-transmit and single-receive antenna aided system seen in Figure 1, when the source data file to be transmitted consists of $K$ number of packets. The LT decoder will require the transmission of $K^{\prime}=K+E$ number of LT encoded packets requiring $E$ redundant packets for the success of the decoding process. After transmission over the BEC imposing an erasure probability of $P_{e},\left(P_{e} \cdot K^{\prime}\right)$ number of the original LT encoded packets will be obliterated.

At the physical layer, we deal with frame-by-fame transmission. Each transmission frame consists of a number of packets. A transmission packet is in turn broken into $S$ number of symbols. Each BICMID source symbol is comprised of $k$ source bits and the BICM-ID encoded symbols have $n=k+1$ encoded bits. The encoded bits are mapped to an $M$-QAM constellation before transmission over an AWGN channel.

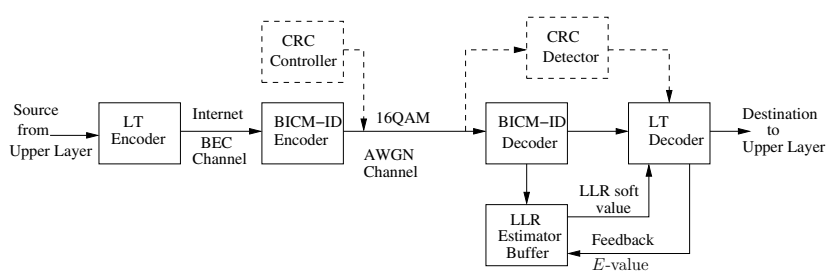

Fig. 1. System structure of the joint design of LT and BICM-ID.

At the receiver seen in Figure 1, BICM-ID decoder receives a prioi information from channel demodulator and output a extrinsic information which is to be fed back to the demodulator for iterative decoding. The sufficiently reliable output bits are then passed to the LT decoder after a number of BICM-ID iterations. We assume here that the perfectly sychronized LT encoder and LT decoder are capable of identifying the indices of the lost packets. The CRC controller inserts CRC bits into the LT encoded packets while the CRC detector extracts the CRC bits and verifies the detectable error inside each packet.

\section{Estimation Scheme}

The LT decoder using the message passing algorithm [1] is inherently sensitive to error propagation. An erroneous packet which contains corrupted bits may propagate the errors from the source nodes of the LT encoder's generator matrix to check nodes, when modulo-2 operations are performed during the message passing decoding [4]. Further related designs were proposed in [5]. By ensuring that a minimum of $E$ redundant packet is available, we can improve the achievable decoding performance by reducing the 
associated error propagation. This can be achieved by discarding erroneous packets, as and when corrupted bits are detected in a packet. Subsequently we employ the CRC-12 code, which is widely used in telecommunication systems for error detection in conjunction with the generator polynomial $x^{12}+x^{11}+x^{3}+x^{2}+x+1$ [6]. This error detection approach improves the system performance but introduces an overhead. Observe in Figure 1 that the CRC controller injects the CRC bits into the BICM-ID encoded 16QAM stream, before transmitting them over the AWGN contaminated BEC. In this paper, we map four bits to the 16 QAM constellation points. The received CRC bits are extracted and are used for detecting the presence of any erroneous bits.

As an alternative, the joint design of LLR-based perfect reliability estimation using BICM-ID has the ability to further improve the system's performance. This potentially allow us to eliminate the CRC overhead bits, while maintaining reliable detection especially at a high SNR. As seen in Figure 1, the soft LLR output of the BICMID decoder is stored in the LLR estimator's buffer. The BICM-ID decoder will set a threshold value for the LLRs, in order to determine which LT packet may contain erroneous bits. Based on their LLR values, each of the corresponding bit is flagged with a '0' or ' 1 ' to indicate a reliable or unreliable bit, which allows the LT decoder to mitigate the associated error propagation effects during the decoding process. A feedback signal is provided by the LT decoder to control the LLR estimator's threshold for the sake of achieving the highest possible detection accuracy.

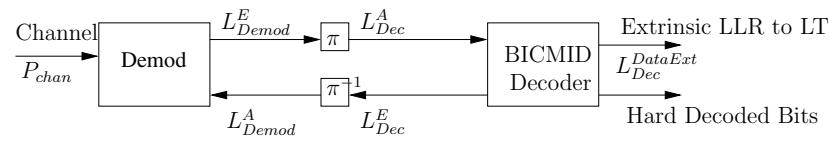

Fig. 2. BICM-ID system structure

Figure 2 shows the basic BICM-ID scheme, where $\pi$ and $\pi^{-1}$ denote the interleaver and deinterleaver. The channel's output information is passed to the demodulator, which receives the a priori LLRs $L_{\text {Demod }}^{A}$ from the BICM-ID decoder and provides the extrinsic LLRs $L_{D e m o d}^{E}$ for the decoder. The channel decoder of Figure 2 receives the a prioiri LLRs $L_{D e c}^{A}$ from the demodulator and outputs the extrinsic LLRs $L_{D e c}^{E}$ in order to perform iterative decoding by exchanging the extrinsic information between the BICM-ID decoder and demodulator. The reliability of the demodulator decisions depends on the channel's Symbol-to-Noise Ratio SNR and on the a priori information $L_{D e m o d}^{A}$, yielding $L_{\text {Demod }}^{E}=f\left(L_{\text {Demod }}^{A} \mid S N R_{\text {chan }}\right)$. By contrast, the reliability of the channel decoder's decisions depends solely on the a priori LLRs received from the demodulator, since it only has a single input and hence it is not directly dependent on the SNR [7], i.e. the extrinsic mutual information $I_{D e c}^{E}=f\left(I_{D e c}^{A}\right)$ and $I_{D e c}^{\text {DataExt }}=g\left(I_{D e c}^{A}\right)$. Using the technique proposed in [7] we are capable of plotting both the EXIT chart and the corresponding BER values are plotted in Figure 3 assuming that the variance of the Gaussian distributed of the decoder output $\sigma^{2}$ is known [7]. The system parameters used in this paper are detailed in Table I.

\begin{tabular}{|l||r|}
\hline BICM-ID code rate & $R=3 / 4$ \\
Modulation & $16 \mathrm{QAM}$ \\
Number of source packets & 10000 \\
Number of transmitted packets & 13000 \\
Number of bits per source packet & 165 \\
BEC erasure probability & $P_{e}=0.1$ \\
LT degree of distribution & Improved Robust Dist. (IRD) \\
\hline \multicolumn{2}{c}{ TABLE I } \\
\multicolumn{2}{c||}{ SYSTEM PARAMETERS. }
\end{tabular}

More explicitly, Figure 3 details the EXIT characteristics of both the demodulator and of the BICM-ID decoder. The nine dotted lines

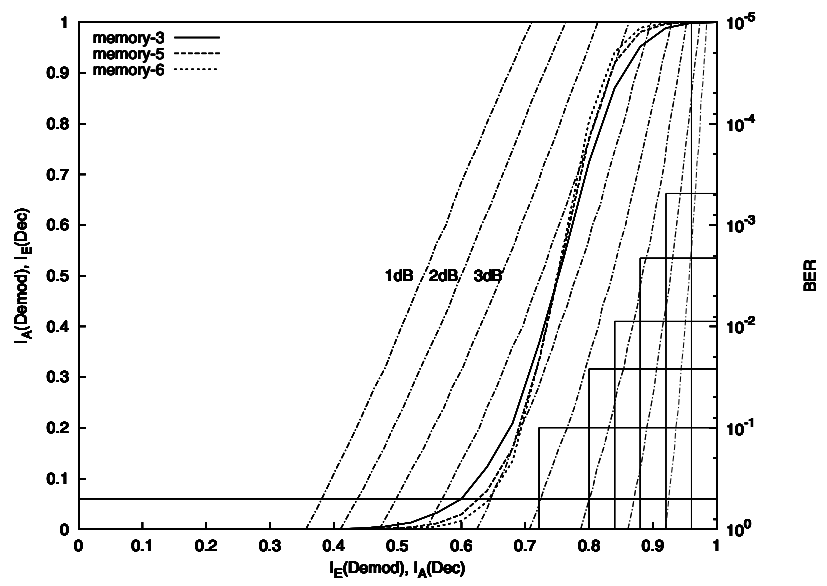

Fig. 3. EXIT chart for BICM-ID.

represents the EXIT characteristics of the demodulator for $E_{b} / N_{0}$ values ranging from $1 \mathrm{~dB}$ to $9 \mathrm{~dB}$ using Ungerböck's Set-Partitioning (SP) mapping strategy. The extrinsic information characteristics for BICM-ID having a coding rate of $R=3 / 4$ and employing different memory lengths of $m=3,4,5$ are shown by the $I_{A}(D e c)$ versus $I_{E}(D e c)$ curves of Figure 3. The BER of the decoder is plotted in the same figure with the aid of the solid horizontal and vertical lines. More explicitly, since over an AWGN channel there is an unambiguous one-to-one relationship between $I_{D e c}^{A}$ and the BER, the output characteristic of the BICM-ID decoder is independent on the channel SNR, the BER curve is based on the function of $P_{b}=f^{\prime}\left[I_{A}(D e c)\right]$ as detailed in [7]. For further illustration, at $I_{A}(D e c)=0.800083$ for example, we have $\mathrm{BER}=0.026177$, as shown in Figure 3.

The threshold value used by the feedback link from the LT decoder to the LLR estimator buffer provides of Figure 1 determine the minimum nuimber of redundant packets required for successful LT decoding. In our fouthcoming discourse we attempt to characterize the intricate interplay between the LT code's generator matrix employing different degree of distributions, the specific portion of redundant packets providing vital information for the LLR estimation buffer in order to adjust its LLR threshold value. Owing to lack of space we simply state that the Improved Robust Distribution (IRD) having parameters of $c=0.1$ and $\delta=0.5$ [4] was used, which required 10 percent redundant packets [4].

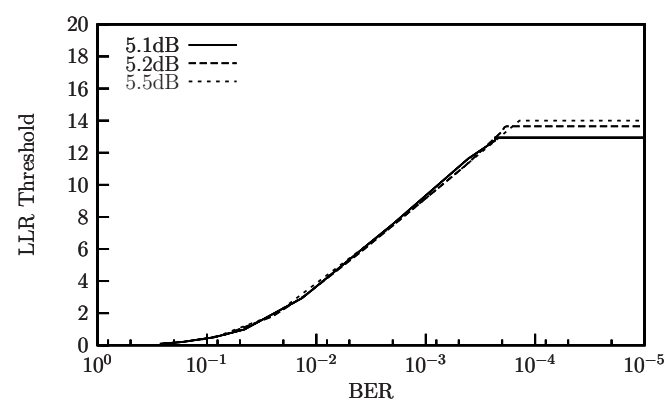

Fig. 4. LLR threshold value necessitated for requiring $E=10 \%$ redundant packets for the system of Table I. The required values were averaged over $165 \mathrm{bit} /$ packet with a total of 10000 LT source packets.

Based on the system parameters listed in Table I, we conducted a series of simulation in order to determine the LLR threshold required for operation at certain BERs at the targeted $E_{b} / N_{0}$ value. Figure 4 shows the LLR threshold value determined by simulation for maintaining a normalised $E$-value of 0.1 at $E_{b} / N_{0}=5.1 \mathrm{~dB}, 5.2 \mathrm{~dB}$ and $5.5 \mathrm{~dB}$, respectively. The horizontal section of the curves represents 
the specific extrapolation of LLR threshold values, when the $10 \%$ target value of $E$ has been reached. The LLR-based packet reliability become sufficiently confident, when the BER of the decoder becomes as low as $2 \times 10^{-4}$.

In Figure 5 the LLR values are plotted for 200 bits against the bitindex taken from a random sample. The entire sample has a total of $165 \times 13000 \times(1-0.1)=1930500$ bits based on Table I. The circles denote the correctly decoded bits, while the crosses denote the erroneously decoded bits. Note that most of the bits that are located outside the threshold region represented by the horizontal lines are indeed likely to be error-free, since at the BER of $2 \times 10^{-4}$ on average a 20000 bit segment would have a single error. By contrast, all the corrupted bits tend to be inside the threshold region, even though some LLR values within the same region may also yield correct bits.

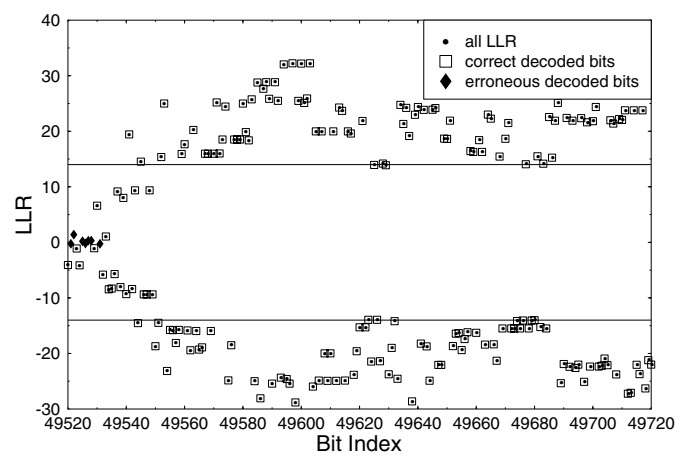

Fig. 5. A set of 200 LLR values recorded for the system detailed in Table I, given the threshold value of Figure 4 at $E_{b} / N_{0}=5.5 \mathrm{~dB}$.

Since we require a BER between $10^{-3}$ to $10^{-4}$ in order to obtain a confident LLR based packet-reliability decision we observe in Figure 3 that the BER of $3 \times 10^{-4}$ satisfies this requirement at $I_{A} \approx 0.92$. This may also be associated with $E_{b} / N_{0}>5.0 \mathrm{~dB}$ for four or more iterations.

\section{LT DeCODING SCHEME}

\section{A. Bit-by-bit LT Decoding}

It is widely recognized that LT packet decoding is sensitive to error propagation. Since it was originally designed for the BEC channel, the BER of the AWGN-contaminated packets may be mitigated by the BICM-ID decoder. An unsuccessfully decoded BICM-ID symbol is likely to cause the error propagation to other LT packets. If the BEC's erasure probability $P_{e}$ is high and the number of redundant LT packets is insufficient, the LT packet decoding operation will be curtailed. This problem can be mitigated by using bit-by-bit LT decoding. With the intrinsically inherited LLR estimation, our detection of corrupted bits can be implemented directly on this new bit-by-bit scheme. The disadvantage is the increase in decoding complexity which is proportional to the number of bits in a packet.
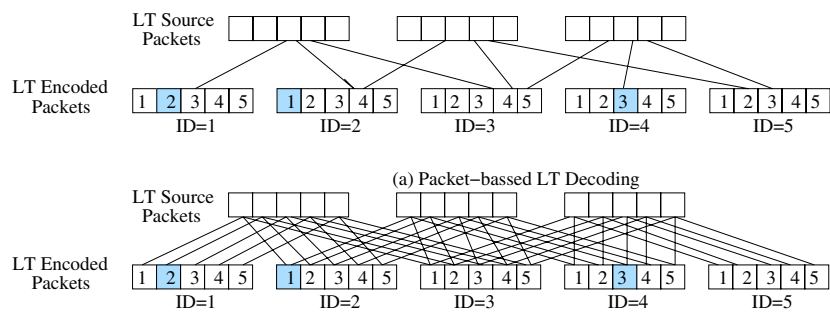

(b) Bit-by-bit LT Decoding

Fig. 6. Packet-based LT decoding and bit-by-bit LT decoding.
Figure 6 illustrates (a) our packet-based LT-decoding and (b) bitby-bit LT-decoding strategies. The corrupted bits of the packets are shown as filled rectangles in the figure.

When using bit-by-bit decoding, the corrupted bits can be identified with the aid of LLR estimation and this enables us to use the rest of the reliable LT-encoded bits and hence to continue the LT decoding process.

\section{B. Pseudo Random LT Generator Matrix}

The design of LT codes critically depends on their so-called degree distribution [8]. It was also stated in [4], [9] that the specific degree distribution of LT codes has a strong influence on the percentage of redundant packets required for error-free detection, which is the $E$-value of the buffer feedback link of Figure 1. Given a specific LTcode degree distribution, a further influential factor in determining the performance of a LT code is the particular choice of the random generator, which controls the actual assignment of the set of original source packets contributing to a specific LT encoded packets, as shown in Figure 6. Since this problem has not been addressed in the open literature, we propose a novel technique for reducing the minimum required fraction of redundant packets in the context of LT codes.

The conventional technique of generating pseudo-random integers for pin-pointing the particular original information packets to be combined with the aid of modulo- 2 additions to generate a given LT-encoded packet is using finite-length Shift Registers (SR). Given an $m$-stage $\mathrm{SR}$, the number of possible integer numbers that may be generated is $M=\left(2^{m}-1\right)$ and the next integer number $I_{n}$ at the output of the SR is uniquely determined by the previous integer number $\left(I_{n}-1\right)$ according to the function of $I_{n}=f\left(I_{n}-1\right)$, which map the finite set $\{I\}$ onto itself. More explicitly, the output of the SR traverses from $I_{n-1}$ to $I_{n}$ by picking any of the $M=\left(2^{m}-1\right)$ elements with the same probability.

As an alternative, a sequence of integer numbers may be generated by linear congruential generators which obey the following relation

$$
I_{j+1}=\left(a I_{j}+C\right) \bmod M,
$$

where $M$ is the modulus, while $a$ and $C$ are positive integers refered to as the multiplier and the increment respectively. The period of the shift register is not longer than $M$. When $M$ is close to its maximum legitimate value of $\left(2^{m}-1\right)$, the sequence generated becomes more correlated, i.e. loses its pseudo-random nature. As a further feature, the linear congruential generator makes the least significant bits more correlated than the most significant bits.

The random integer number generator used for LT codes in [8] [10] and [4] uses the random generator which has the length of $2^{32}$ equal to a 32 bits register and the process is based on the formula

$$
I_{n}=\left(I_{n-1}+C\right) \bmod M
$$

We opted for using the random sequence generated from (2) for LT encoding, when choosing randomly the modulo- 2 connections between the LT source packets and the LT encoded packets as illustrated in Figure 6. An example of the degree of connections between the LT source and LT-encoded packets is shown in Figure 7, corresponding to $1.06 \times 2011$ LT-encoded packets, where the multiplier 1.06 indicates that $6 \%$ redundant packets were generated.

Note that from Figure 7 there are some indices of LT packets, which are not selected after the random selection. Their value are zeros as shown in Fig. 7. This means these LT packets are still not processed in the LT encoding, which causes the failure in the LT decoding process to recover all source LT packets. In this case, to ensure all source packets are collected we need to increase the number of output encoded LT packets. It means we have to increase the 


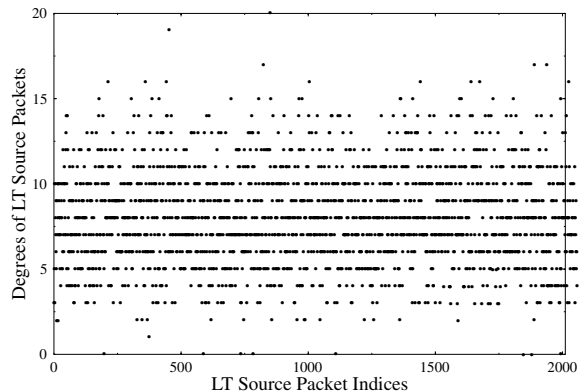

Fig. 7. A snapshot of the integer random numbers generated by the congruential generator of (2) for $M=32$.

overhead of the LT encoded packets. For the sake of avoiding this, we introduce a solution for designing a better random integer number generator of LT codes.

For the sake of generating an uncorrelated set of integer numbers we apply the random generator relying on rearranging the bits of a shift register proposed in [3] [11]. We introduce two different types of random rotation generators. The first method suggests that the bits are rotated after their modulo 2 addition

$$
I_{n}=\left(\left(I_{n-j}+I_{n-k}\right) \bmod 2^{b}\right) \operatorname{rot} r .
$$

By contrast, the second method proposes that the bits are rearranged before their modulo 2 addition

$$
I_{n}=\left(\left(I_{n-j} \operatorname{rot} r_{1}\right)+\left(I_{n-k} \operatorname{rot} r_{2}\right)\right) \bmod 2^{b},
$$

where $I_{n}$ is an integer presented by $b$ bits and the notation $I_{n-j}(r o t) r_{1}$ means that the bits of $I_{n-j}$ are shifted to the right by $r_{1}$ positions, for example according to $00001111_{2}(\mathrm{rot}) 3=11100001_{2}$. When using the pseudo-random integer number generator of (4), the LT code achieves a better performance than using the traditional linear congruential pseudo-random integer number generators of (2) although these results are not included here owing to lack of space. Fig. 8 shows the evolution of the degree of connections for the LT source packet indices after encoding 1.06x2011 number of LT encoded packets using the pseudo-random integer number generator of (4), where in contrast to Figure 7 no degree-zero connections are found, which can cause potential decoder failures.

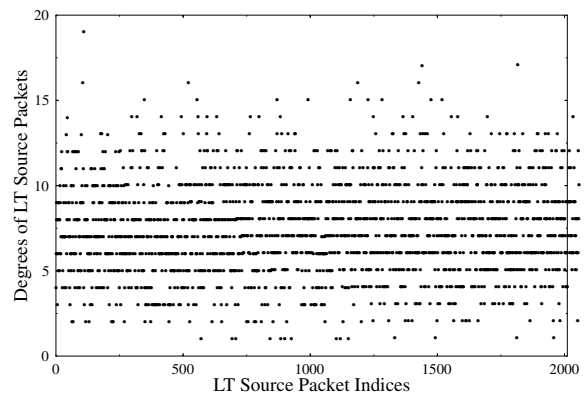

Fig. 8. A snapshot of the integer random numbers generated by the pseudorandom generator of (4).

We further investigate the benefits of the specific pseudo-random generator of (4) by comparing the normalised number of packets required for successful LT decoding. The parameters of the specific LT degree of distribution used in the simulations are $c=0.1, \delta=0.5$ [4] and the required number of LT encoded packets is normalized by the number of LT source packets $K$. From Figure 9 and Figure 10 we observe that the number of LT encoded packets required in Figure 9 is smaller than in Figure 10. More specifically, the required number of LT encoded packets only varies in the range spanning from 1.06 to 1.085 in Figure 9, while it varies from 1.06 to 1.10 in Figure 10.

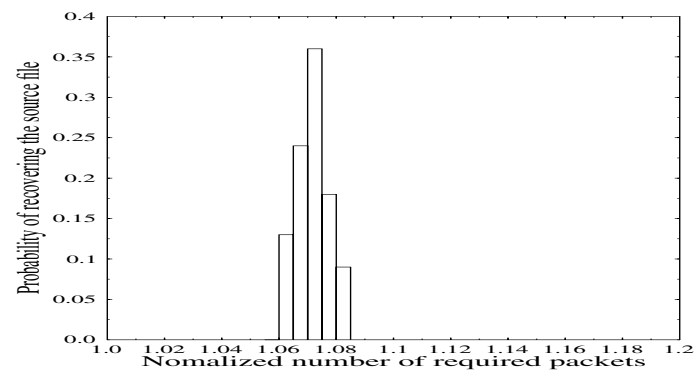

Fig. 9. The degree histogram of the LT code using the pseudo-random integer number generator of (4)

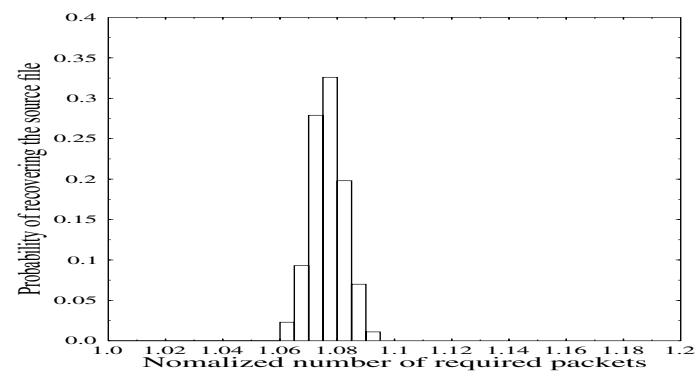

Fig. 10. The degree histogram of the LT code using the linear congruential pseudo-random integer number generator of (2).

\section{Simulation Results}

In this section we embark on quantifying the simulation results for our proposed joint scheme. The system parameters employed in our simulation results are shown in Table I.

When encoding the same number of source packets $K=2011$, Figure 11 shows the BER versus the normalized number of LTencoded packets using both the pseudo-random and congruential pseudo-random integer number generators of (2) and (4). It can be seen from Figure 11 that the random generator of (4) reduces the total number of LT-encoded packets compared to that of (2) by $3 \%$ at $\mathrm{BER}=10^{-5}$.

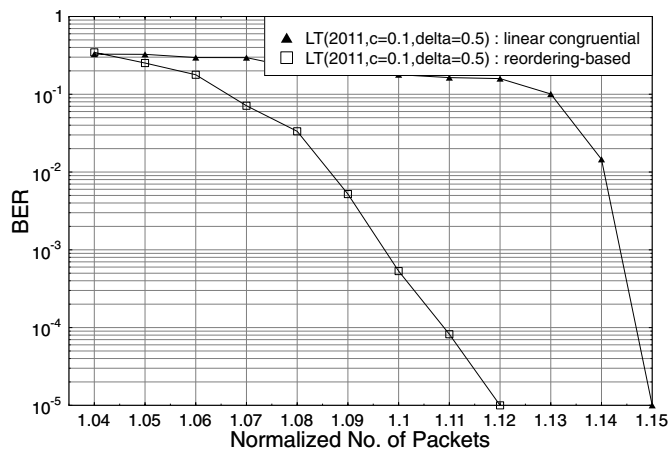

Fig. 11. BER versus the number of LT-encoded packets when transmitting 2011 packets over a BEC having $P_{e}=0.1$ and contaminated by AWGN in conjunction with the system parameters of Table I and using $c=0.1, \delta=0.5$.

The BER performance of the BICM-ID scheme using different number of decoding iterations combined with LT coding is shown in Figure 12 in comparison to that of our benchmark scheme operating without any exchange of information between the BICM-ID and LT 


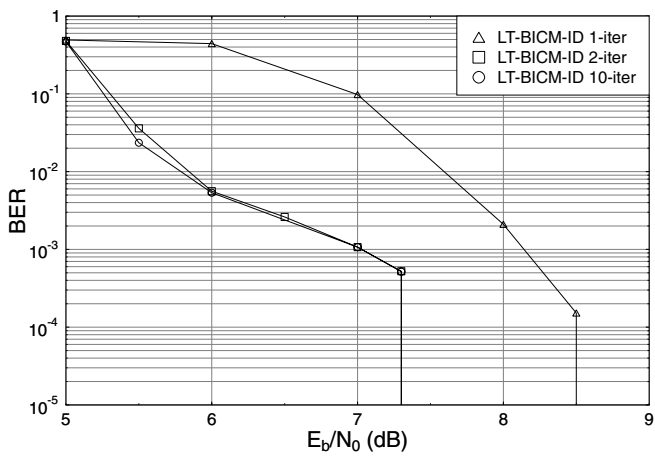

Fig. 12. BER versus $E_{b} / N_{0}$ performance, when communicating over a BEC channel having $P_{e}=0.1$ and contaminated by AWGN, when using thedegree distribution of (4).

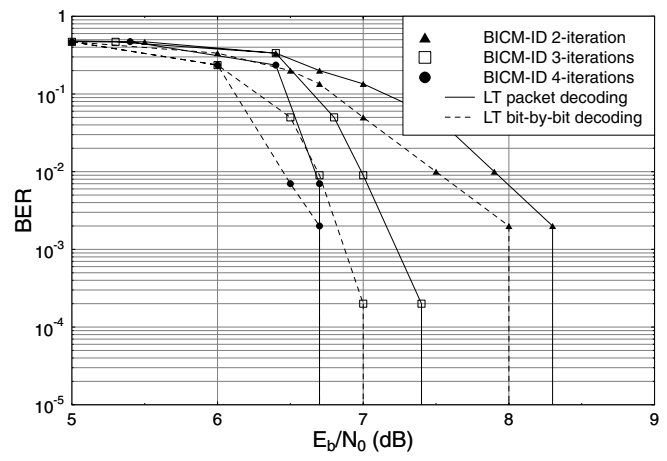

Fig. 13. BER versus $E_{b} / N_{0}$ performance, when communicating over a BEC channel having $P_{e}=0.1$ contaminated by AWGN using perfect CRC estimation as well as the parameters of Table I.

decoder. An infinitesimally low BER is achieved for $E_{b} / N_{0}$ values in excess of $7.4 \mathrm{~dB}$.

When using an exchange of soft information between the LLR buffer and LT decoder of Figure 1, Figure 14 shows the significant performance improvement achieved by the system. Figures 13 and 14 include the results recorded for both the LT packet decoding as well as for the LT bit-by-bit decoding methods.

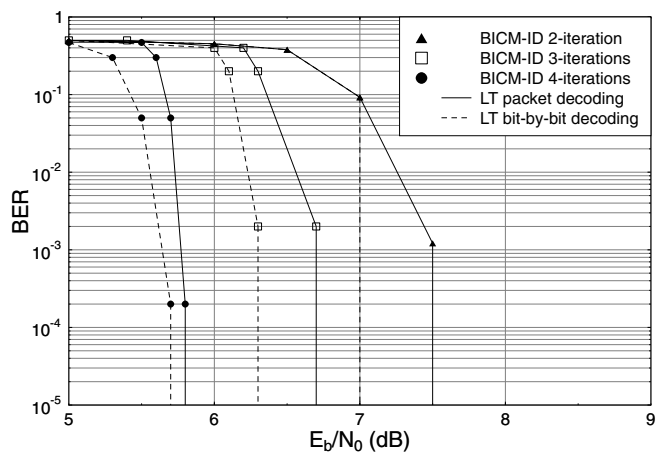

Fig. 14. BER versus $E_{b} / N_{0}$ performance, when communicating over a BEC channel having $P_{e}=0.1$ and contaminated by AWGN using LLR based reliability estimation and the parameters of Table I.

Finally, in Figure 15 we compare the bit-rearrangement based pseudo random generator of (4) and the traditional congruent random generator of (2) for specifying the modulo- 2 connections between the LT source packets and the LT-encoded packets. The technique of (4) is capable of reducing the number of source packets required for achieving infinitesimally low BER. More explicitly, Figure 15 shows the improved BER performance recorded, when using $K=11000$ packets instead of the originally stated $K=13000$ packets specified in Table I. This is achieved when the available redundant packets have been limited by the source input.

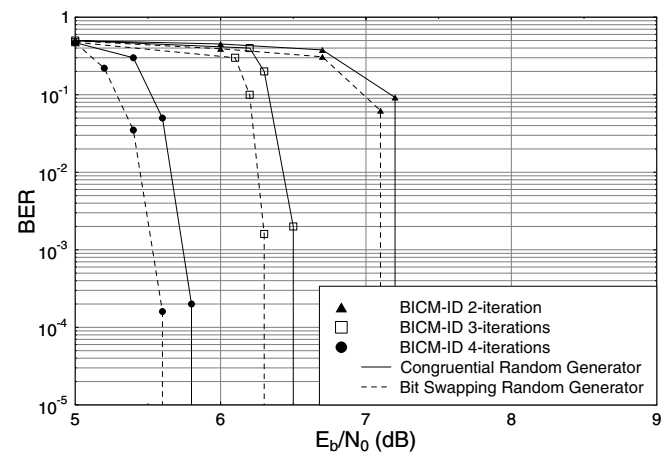

Fig. 15. BER versus $E_{b} / N_{0}$ performance when communicating over a BEC channel having $P_{e}=0.1$ and contaminated by AWGN using LLR based reliability estimation and the parameters of Table I. The number of source packets was reduced to $K=11000$ from $K=13000$

\section{CONCLUSIONS}

A jointly designed BICM-ID and LT coding scheme was proposed for mitigating the effects of the AWGN-contaminated BEC. The novel features of the scheme included:

1) a bit-by-bit LT decoder;

2) an LLR-based reliability estimator for the sake of avoiding the classic CRC overhead;

3) a bit-rearrangement based random generator, which resulted in an improved LT-generator, which is capable of reducing the LT-encoded packet overhead required for maintaining an infinitesimally low BER.

\section{REFERENCES}

[1] J. Buyers, M. Luby and M. Mitzenmacher, "A Digital Fountain Approach to Asynchronous Reliable Multicast," IEEE Journal on Selected Areas in Communications, vol. 20, pp. 1528-1540, October 2002.

[2] E. Zehavi, "8-PSK Trellis Codes for a Rayleigh Fading Channel," IEEE Transactions on Communications, vol. 40, pp. 873-883, May 1992.

[3] M. Matsumoto and T. Nishimura, "A 623-Dimensionally Equidistributed Uniform Pseudo-Random Number Generator," ACM Transactions on Modeling and Computer Simulation, vol. 8, pp. 31-42, 1998.

[4] R. Y. S. Tee, T. D. Nguyen, L. L. Yang and L. Hanzo, "Serially Concatenated Luby Transform Coding and Bit-Interleaved Coded Modulation Using Iteratlive Decoding for the Wireless Internet," in Vehicular Technology Conference, vol. 5, (Melbourne, Austrialia), pp. 2494-2498, Spring 2006.

[5] T.D. Nguyen. F.C. Kuo, L-L. Yang, L. Hanzo: Amalgamated Generalized Low Density Parity Check and Luby Transform Codes for the Wireless Internet, Proceedings of IEEE VTC'07 Spring, April, 2007, Dublin, Ireland, pp 2440 - 2444

[6] Q. Luo and P. Sweeney, "Hybrid-ARQ Protocols Based on Multilevel Coded Modulation," IEE Electronics Letters, vol. 39, pp. 1063 - 1065, July 2003 .

[7] T. Clevorn, S. Godtmann and P. Vary, "BER Prediction Using EXIT Charts For BICM With Iterative Decoding," IEEE Communications Letters, vol. 10, pp. 49-51, January 2006.

[8] M. Luby, "LT codes," in Proceeding of the 43rd Annual IEEE Symposium on Foundations of Computer Science, pp. 271-282, November 2002.

[9] D. J. C. MacKay, Fountain Codes. http://www.inference.phy.cam.ac.uk/ mackay/CodesTheory.html: Cavendish Laboratory, University of Cambridge, 2004.

[10] D. J. C. MacKay, Information Theory, Interference and Learning Algorithms. http://www.inference.phy.cam.ac.uk/mackay/itila/: Cambridge University Press, 2003.

[11] A. Fog, Chaotic Random Number Generators with Random Cycle Lengths. http://www.agner.org/random/theory, 2004. 